\title{
Medicina comunitaria. Introducción a un análisis crítico
}

\author{
Community medicine. \\ Introduction to a critical analysis
}

Jairnilson Silva Paim ${ }^{1}$

${ }^{1}$ Médico. Doctor en Salud Pública. Profesor Titular de la Universidad Federal de Bahía, Brasil. jairnil@ufba.br
RESUMEN Este artículo de Jairnilson Silva Paim, publicado originalmente en 1976 en la revista Saúde em Debate del Centro Brasileiro de Estudos de Saúde (CEBES), realiza un análisis del concepto "comunidad", como así también de la utilización de las expresiones "salud comunitaria" y "medicina comunitaria" relacionadas a la propuesta de medicina integral y medicina preventiva. Su publicación en español en la sección Textos fundacionales, apunta a la reconstrucción histórica de un movimiento originado en América Latina como medicina social y/o salud colectiva hace más de cuatro décadas y que en el caso de Brasil tiene como claros antecedentes la obra de Sergio Arouca O Dilema preventivista: contribuição para a compreensão e crítica da medicina preventiva y las de Cecília Donnangelo Medicina e sociedade: o médico e seu mercado de trabalho y Medicina e estrutura social: o campo da emergência da medicina comunitária. Estos tres textos son producto de tesis de doctorado y libre docencia, escritos en el primer lustro de los años '70 al igual que este artículo que estamos publicando, y otros que el CEBES recupera en su número especial del año 2008, titulado Saúde em Debate: Fundamentos da Reforma Sanitária.

PALABRAS CLAVE Medicina Comunitaria; Políticas Públicas de Salud; Salud Colectiva; Medicina Social.

ABSTRACT This article by Jairnilson Silva Paim, originally published in 1976 in the journal Saúde em Debate belonging to the Centro Brasileiro de Estudos de Saúde (CEBES), makes an analysis of the concept of "community", together with the use of the expressions "community health" and "community medicine" related to the proposal of integral medicine and preventive medicine. Its publication in Spanish in the Founding Texts section points at the historical reconstruction of a movement originated in Latin America as social and/or collective medicine more than four decades ago. In the case of Brazil, it has, as clear antecedents, the work of Sergio Arouca O Dilema preventivista: contribuição para a compreensão e crítica da medicina preventiva and Cecília Donnangelo's ones Medicina e sociedade: o médico e seu mercado de trabalho and Medicina e estrutura social: o campo da emergência da medicina comunitária. These three texts are the product of a PhD. thesis and free teaching, written in the early seventies as well as the articles we are publishing and others that the CEBES recovers in its 2008 special issue with the title Saúde em Debate: Fundamentos da Reforma Sanitária.

KEY WORDS Community Medicine; Health Public Policy; Collective Health; Social Medicine. 


\section{INTRODUCCIÓN}

El análisis de los temas "salud comunitaria" y "medicina comunitaria" inicialmente presenta ciertas dificultades semánticas, dado que esas expresiones han sido usadas de manera corriente como sinónimos de "salud pública", de "medicina social", de "medicina integral" o incluso de "medicina preventiva" (1-4). Por otro lado, el uso indiscriminado de esas expresiones, además de propiciar la aparición de problemas conceptuales y operacionales, excluye la perspectiva de identificar las diferentes situaciones históricas que desencadenaron esos movimientos ideológicos.

La idea de comunidad ha sido fuertemente introducida en el pensamiento y la actividad profesional en los últimos tiempos y según Ferrara et al. (2) a través de ella se ha jerarquizado la función social de la medicina; aunque valdría la pena indagar cuál es el concepto de "comunidad" que estaría siendo usado por los diferentes autores. Es posible incluso que al observar ciertos programas de salud comunitaria lleguemos a la conclusión de que ellos no sean ni de salud ni de comunidad.

Más allá de que un abordaje sociológico sobre el tema "comunidad" escape al objetivo inmediato del presente trabajo, es interesante discutir brevemente la evolución de este concepto.

\section{ORÍGENES DE LA MEDICINA COMUNITARIA}

Aunque los aspectos históricos de la medicina comunitaria aún no hayan sido debidamente estudiados, se la podría identificar como originaria de EE.UU., especialmente a partir de la década del '60. Desde esa perspectiva, la medicina comunitaria sería visualizada bajo dos vertientes principales (5):

a) ejecutada por agencias voluntarias u órganos estatales, apuntando a llenar las lagunas entre la medicina privada y la medicina pública;

b) articulada a las escuelas médicas, en el sentido de operacionalizar el movimiento de la medicina preventiva.
Se ha resaltado que la divulgación de la práctica de la medicina comunitaria en la década del '60 coincide con la administración Kennedy y su política de "combate a la pobreza" y especialmente a la denominada "nueva ley del pobre", reforzándose con la administración Johnson a través de los programas Medicare y Medicaid. Como forma de acción, se caracterizaba por un trabajo multiinstitucional y multiprofesional actuando especialmente a nivel de los guetos (5).

\section{DISCUSIÓN SOBRE EL CONCEPTO DE "COMUNIDAD"}

El concepto de "comunidad" ha sido abordado tanto como idea estructural (límites geográficos, autonomía administrativa, habitantes comprometidos en actividades interrelacionadas, etc.) como idea de proceso, desarrollada especialmente durante la última mitad del siglo XIX. Según Lindman (6), el sistema industrial se tornó impersonal y se proyectó más allá de los límites de la comunidad; los establecimientos industriales se localizaban en áreas geográficas definidas, pero pertenecían a empresarios que habitaban en otras comunidades; los empresarios ganaban más movilidad, trasladándose más fácilmente de una comunidad a otra; los medios de comunicación se perfeccionaron rápidamente haciendo que la comunidad sea menos autolimitada. Así, las ciencias sociales pasaron a rever sus conceptos y

\footnotetext{
...la dinámica de la sociedad fue vista ya no como fruto de su estructura, sino explicada preferentemente por los intereses, aspiraciones, necesidades y objetivos de los individuos interactuando con otros individuos en la diversidad de los agrupamientos sociales. (6)
}

De este modo, los principales factores condicionantes de la "naturaleza humana" eran considerados de carácter psicológico. Desde esta perspectiva, los individuos interactúan intensamente al ser portadores de valores comunes. En esas interacciones actuarían desempeñando ciertos roles sociales de acuerdo con las expectativas 
de comportamiento establecidas por el grupo. Tales expectativas, a su vez, serían vinculadas a los procesos de socialización y de control. La comunidad podría ser entendida como la sociedad en la que los conflictos fueron eliminados, restando la idea de sociedad a la sumatoria de comunidades. No obstante, si "comunidad" es considerada como un proceso, al describir el modo en que los seres humanos interactúan ¿por qué no es sinónimo del concepto de "sociedad"? (6).

De este modo, algunos autores pasaron a usar el término "comunidad" en el sentido de "sociedad", mientras que otros

...restringían el término a los aspectos más positivos de la interacción social, o sea, para designar todas las formas de asociación en las cuales el conflicto superfluo fue eliminado.

Para Lindman (6), una definición válida incluiría tanto el elemento estructural como el funcional:

Cualquier agrupamiento de individuos concientemente organizados, habitando un área específica o una localidad, dotada de autonomía política limitada, manteniendo instituciones primarias como escuelas e iglesias entre las cuales son reconocidos ciertos grados de interdependencia [o incluso] cualquier proceso de interacción social que genera una actitud y una práctica más intensiva y extensiva de interdependencia, cooperación y unión.

Otros autores defienden la teoría de que la participación en una comunidad deriva de una conciencia de pertenecer (sentido de pertenencia histórica, política y económica) (2).

La crítica realizada a esa concepción estructural-funcional de comunidad apunta al hecho de que subestima, cuando no anula, las posibilidades de cambio; no incluye el concepto de "clases", y sí el de "estratos"; cosifica al hombre (sumisión al rol); se relaciona con la línea de patología social en cuanto a las denominadas conductas desviantes (corriente funcionalista).

\section{ALGUNOS ENFOQUES PARA LA MEDICINA COMUNITARIA}

Según Barton y Smith (1), la medicina comunitaria se interesa por los problemas relacionados con la salud del pueblo, al estar entre la práctica privada de la medicina en el consultorio, la clínica y el hospital y los servicios ofrecidos por las agencias de salud pública. Abarca procedimientos preventivos y curativos dirigidos a las familias o a grupos de la población que no son provistos adecuadamente por otras agencias existentes. Grant (3), en 1967 publicó un libro bajo el título Handbook of preventive medicine and public health, pero luego en la reedición, justificó el cambio del título por Handbook of community health, dado que la expresión "salud comunitaria" le daría un uso más definido y podría tal vez ser considerada más abarcadora que las palabras del título original.

En los países europeos donde prevalecen la medicina estatal y la socializada esta concepción de medicina comunitaria podría parecer incoherente y sin propósito, aunque se observa la utilización de la expresión "medicina comunitaria" (7). Sin embargo se percibe el entendimiento de la medicina comunitaria como una representante avanzada de la salud pública clásica, en la medida en que utiliza conocimientos de otras disciplinas tales como la sociología, la psicología social, la ecología, la genética, etc. De este modo, la medicina comunitaria, como disciplina, posibilitaría al estudiante entender el comportamiento social, en vez de restringirse al estudio del comportamiento de la bacteria (7). Reforzaría por lo tanto el estudio y la aplicación de las ciencias sociales en sus relaciones con la salud humana.

Otros autores $(2,8,9)$ ven la medicina comunitaria como la realización de la medicina integral, mediante la aplicación de las acciones preventivas y curativas del medio ambiente con la participación activa de la comunidad organizada. Según Devoto (9), "solo una participación activa de la comunidad, en una empresa que otorgue salud, en cuyo seno exista un espíritu de comunidad de trabajo, realizado mediante métodos democráticos y de autogestión, podría ser el fundamento de una medicina comunitaria". En esta visión, las acciones integradas de salud, serían 
las medidas de protección, promoción, recuperación y rehabilitación, realizadas por un equipo de salud. Se percibe en este enfoque el énfasis en el equipo de trabajo polivalente -cuyo liderazgo podría caberle o no al médico-, en la necesidad de que el profesional de salud se torne miembro de la comunidad para desarrollar una mayor confianza y aproximación con el pueblo, y en la identificación de líderes (8).

Están los que consideran que "la población deberá comprender su capacidad de presión para obtener beneficios colectivos" (8), o incluso que la medicina comunitaria no debe ser "la ataráxica de la tensión social" (9), pues su finalidad es la participación auténtica y profunda de la comunidad.

De este modo, Devoto (9) discute la viabilidad de la medicina comunitaria en tres vías de desarrollo socioeconómico: capitalismo nacional, capitalismo de Estado y el socialismo desarrollista, en base al esquema propuesto por Jaguaribe (10). Según el autor, en el primer modelo, el concepto de medicina integral puede ser aceptado por los funcionarios estatales y desarrollar una medicina estatal. Si el modelo tiene éxito, la medicina comunitaria se desvirtúa en su esencia, aunque posibilite una medicina social más humana y productiva. Si el modelo fracasa, y se evita el retroceso a formas más regresivas, la medicina comunitaria podría favorecer al pasaje a modelos más concordantes con su espíritu.

En el modelo de capitalismo de Estado, la medicina comunitaria tendría características estatales, pero sin descentralización y sin una mayor autonomía de las unidades locales. Para el autor en cuestión, es difícil dentro de este modelo la participación comunitaria en la estructura estatal, siendo posible una evolución tanto hacia el neocapitalismo como hacia el socialismo desarroIlista. Este tercer modelo impulsaría la industrialización y resultaría del fracaso del capitalismo dependiente, de la no viabilidad de los modelos desarrollistas o de una ulterior evolución del capitalismo de Estado. La perspectiva de una máxima descentralización y autogobierno local con una participación democrática de la comunidad proporcionaría, según el autor, la concretización de la finalidad de la medicina comunitaria. Por último, espera que este enfoque proporcione un marco de referencia conceptual "ligando la medicina social realizada con la participación activa de la comunidad, al cambio y desarrollo".

Si bien se percibe la preocupación de este autor por buscar, en la medicina social y en la medicina integral, los marcos teórico y conceptual para la práctica de la medicina comunitaria, por otro lado se observa que la relación de esa práctica con los modelos de desarrollo descriptos por Jaguaribe (10) se restringe a un plano de abstracción, dado que la historia de América Latina no parece haber producido fiel y mecánicamente aquellas vías políticas de desarrollo económico (11). Así, lo importante sería ver en la práctica cuáles son los objetivos que la medicina comunitaria ha alcanzado (8). Por consiguiente, la práctica de la medicina comunitaria como posibilidad de cambio no parece tener sustento en la experiencia histórica de los países, aunque pueda representar la visión del mundo de este o de aquel autor. Concretamente, sin embargo, podría ser aceptada desde una óptica funcionalista (pequeños obstáculos a ser delimitados por el sector salud) o cuestionada por un enfoque teórico que identifique una matriz determinante más global en la génesis de los problemas de salud (5).

De este modo, el surgimiento de la medicina comunitaria, tanto en los países industrializados como en los países subdesarrollados, podría estar relacionado con la crisis del sector salud observada en ambas categorías de países, aunque con una configuración cualitativamente distinta. En los países latinoamericanos, esta crisis tiene características especiales, como sugirió Arouca (12), pues presenta simultáneamente una "morbilidad moderna" y una "morbilidad de la pobreza", y una yuxtaposición de la incapacidad de solución de problemas básicos, como enfermedades infecciosas y carenciales, con una moderna tecnología de transplantes cardíacos, unidades coronarias, etc. En ese trabajo, el autor Ilama la atención sobre los siguientes puntos:

1. "El sector salud se encuentra en una crisis de realización derivada del bajo impacto y alto costo del actual conocimiento médico."

2. "La medicina contemporánea se caracteriza por la creciente corporificación del conocimiento en tecnología de alta densidad de capital, ejercida por agentes altamente especializados, diversificados y prestigiados, en la 
que cada vez más el acto médico se transforma en la técnica y en el instrumental, provocando una baja cobertura poblacional."

3. "El sector salud posee como factor preponderante el diagnóstico y la terapéutica, y centra sus acciones sobre los "complejos de efectos", produciendo constantemente una población necesitada de servicios médicos."

4. "La enseñanza médica participa de la crisis de la realización del sector en la medida en que continúa formando profesionales con las características anunciadas, pero simultáneamente, define su crisis específica al cuestionar la adecuación de sus graduados a las necesidades de la población. Como consecuencia de este cuestionamiento se crea una crisis ideológica consustanciada en la revisión de los procedimientos educacionales, de evaluación, y del paradigma hospitalario como centro del sector educacional."

Así, la medicina comunitaria, articulada a las escuelas médicas o en tanto funcióntapón entre la medicina privada y la medicina pública, podría ser entendida como un intento idealista de superar los impasses en que se encuentra el sector salud.

\section{ALGUNAS PREGUNTAS SOBRE LA MEDICINA COMUNITARIA}

Al hablar de medicina comunitaria, de inmediato se plantean algunas preguntas. ¿La comunidad sería el objeto de trabajo de la medicina comunitaria? ¿O serían las poblaciones periféricas, las de bajos ingresos, la marginalidad urbana, el ejército industrial de reserva o incluso la sociedad civil?

De este modo, la cuestión de la comunidad y a su vez la de la medicina comunitaria pasa a relacionarse con el modo de producción, el modelo político, la marginalidad urbana, la industrialización, las migraciones, la urbanización, el desarrollo/subdesarrollo, y particularmente con el crecimiento económico acelerado y la distribución de renta.

En este contexto, la medicina comunitaria estaría atendiendo las siguientes proposiciones: a. como operacionalidad del concepto de "medicina integral" en la formación de los recursos humanos para la salud;

b. como posibilidad de experimentación de modelos de prestación de servicios de salud, asimilados o no por el sistema de atención médica;

c. como forma de Ilenar lagunas entre la medicina privada y la medicina pública;

d. como la acción que procede del Estado en el sentido de modernizar la concepción de salud de la población;

e. como posibilidad de intervención en el sentido de dirigir los hábitos, actitudes y comportamientos de la población hacia fines definidos por sectores dominantes, o sea, el control social, desde la domesticación de la puericultura y de la higiene mental hasta las formas más declaradas de control de la natalidad.

\section{CONCLUSIONES}

En la medida en que la medicina comunitaria, en tanto práctica, se valió del movimiento ideológico de la medicina integral, incorporó naturalmente en su acción el énfasis en lo profesional (visión amplia de la problemática de la salud, liderazgo, articulación de agencias, habilidad técnica, actuación centrada en el paciente, etc.) y en la enseñanza (extrahospital universitario, y con la inclusión de otras disciplinas, tales como sociología, psicología social, antropología, etc.).

En consecuencia, sus proposiciones no exigirían modificaciones relevantes en la organización del sistema de atención médica, especialmente en lo referente a las contradicciones entre los subsistemas de producción de los servicios público y privado. Tal como la medicina preventiva:

...como esfuerzo por preservar la medicina liberal como una institución social, refleja menos un instrumento favorable a los cambios necesarios que un intento de preservación del orden existente, no solo en el campo de la atención médica y de la salud, como en el de la organización profesional. (13) 
No obstante, la reflexión teórica impuesta a los trabajadores del sector salud por la crisis actual, puede ayudarlos a dar el salto cualitativo desde una conciencia ingenua hacia una conciencia crítica y a proponer soluciones menos parciales para los problemas de salud de las poblaciones.

\section{NOTAS FINALES}

a. Este texto fue publicado originalmente en el primer número de Saúde em Debate, en el año 1976, bajo el título Medicina comunitária. Introdução a uma análise crítica. Fue reproducido posteriormente en el 2008, como facsímil del original, en la compilación organizada por Sonia Fleury, Ligia Bahia y Paulo Amarante que Ileva por título Saúde em Debate: fundamentos da Reforma Sanitária, editado por el Centro Brasileiro de Estudos de Saúde (CEBES). Agradecemos al CEBES y en especial a Sonia Fleury, por autorizar su reproducción.

\section{REFERENCIAS BIBLIOGRÁFICAS}

1. Burton LE, Smith HH. Public health and community medicine. 2da. Ed. Baltimore: Williams and Wilkins; 1975.

2. Ferrara FA, Acebal E, Paganini SM. Medicina de la comunidad. Buenos Aires: Inter Médica Editorial; 1972. p. 52-54.

3. Grant M. Handbook of community health. 2da ed. Filadelfia: Lea \& Febiger; 1975.

4. Porterfield JD, organizador. Saúde comunitária nos Estados Unidos. Río de Janeiro: Livraria Freitas Bastos; 1972.

5. Relatório da Reunião do Grupo de Estudo e Pesquisa em Saúde Mental da UFBA. Salvador de Bahía: UFBA; 1976. (Mimeo).

6. Lindman EC. Community. En: Seligman ERA, Johnson A, editores. Encyclopedia of the social sciences. Nueva York: MacMillan Company; 1951.
7. Nelson AM. A handbook of community medicine. Bristol: John Wright and Sons Limited; 1975.

8. Centro de Estudos de Saúde da UFMG. Medicina comuitária. Nêmesis. 1975;(1):35-42.

9. Devoto E. La medicina comunitaria y las vías de desarrollo socioeconómico. Cuadernos de Medicina Social. 1970;11(3);33-41.

10. Jaguaribe H. Desarrollo económico y desarroIlo político. Buenos Aires: Eudeba, 1964.

11. Cardoso FH. O modelo político brasileiro. 2da. ed. San Pablo: Difusão Européia do Livro; 1973. p. 8-13, 58-71.

12. Arouca $\mathrm{S}$. Introdução à crítica do setor saúde. Nêmesis. 1975;(1):17-24.

13. Departamento de medicina preventiva da USP. Medicina preventiva e níveis de saúde das populações. Curso Experimental de Medicina. San Pablo: USP; 1970. (Mimeo).

\section{FORMA DE CITAR}

Silva Paim J. Medicina Comunitaria. Introducción a un análisis crítico. Salud Colectiva. 2009;5(1):121-126. 